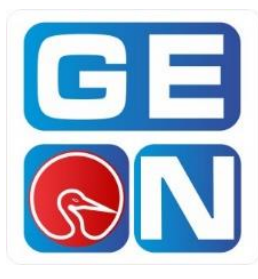

Revista GEON (Gestión, Organizaciones y Negocios.) ISSN: 2346-3910 en línea

revistageon@unillanos.edu.co

Universidad de los Llanos

Colombia
Álvarez Hernández, Luz Angélica; Navarro Quintero, Dora;
Montoya, Aida Viviana; Gil Berrio, Andres.
Modelos de gestión organizacional y planeación estratégica de las clínicas odontológicas especializadas
ODONTOSTUDIO limitada
Revista GEON, Vol. 5, No. 2, 2018
Pág. 38-49

Disponible en: $\underline{\text { https://doi.org/10.22579/23463910.32 }}$

Esta publicación se encuentra bajo licencia: Creative Commons

ReconocimientoNoComercial-

SinObraDerivada 4.0 Internacional

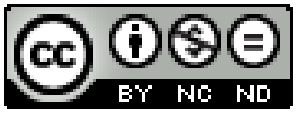

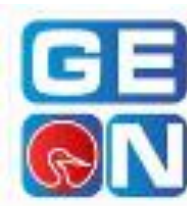
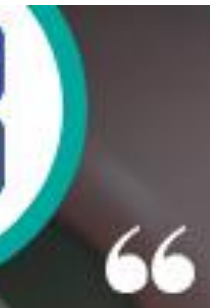

El análisis de la situación de la empresa ODONTOSTUDIO;

identifica una organización sólida y definida,en cuanto a gestión humana, estrategias, cultura

organizacional

RevistaGEON in $9 f$ int 


\title{
Modelos de Gestión Organizacional y Planeación Estratégica de la Clínicas Odontológicas Especializadas ODONTOSTUDIO Limitada
}

\section{Models of Organizational Management and Strategic Planning of the Specialized Dental Clinics ODONTOSTUDIO Limitada}

\author{
Luz Angélica Álvarez Hernández \\ luz.alvrezh@campusucc.edu.co \\ Administradora de Empresas, Universidad Cooperativa de Colombia, sede Villavicencio, Colombia, \\ @ angelic299
}

\section{Dora Navarro Quintero \\ dora.navarro@campusucc.edu.co}

Administradora de Empresas, Magister en Ciencias de la Educación, Universidad Cooperativa de Colombia, sede Villavicencio, Colombia

\section{Aida Viviana Montoya \\ aida.montoyam@campusucc.edu.co}

Administradora de Empresas, Universidad Cooperativa de Colombia, sede Villavicencio, Colombia, @nani_tha17

\section{Andres Gil Berrio \\ andres.gilb@campusucc.edu.co}

Administrador de Empresas, Universidad Cooperativa de Colombia, sede Villavicencio, Colombia, @ andresgil1019

Revista GEON Vol. 5 No 2 julio-diciembre 2018

ISSN 2346-3910 en línea http://revistageon.unillanos.edu.co/index.php/geon/article/view/32 


\title{
Resumen
}

En el actual contexto de globalización, las empresas deben estar a la vanguardia de los cambios organizacionales para mejorar la productividad y competitividad. El propósito de la investigación aplicada es comparar los resultados de la matriz de diagnóstico del Modelo de Modernización para la Gestión de las Organizaciones (MMGO) con los resultados de la matriz de diagnóstico Debilidades, Oportunidades, Fortaleza, Amenazas (DOFA) de la empresa prestadora de servicios odontológicos, ODONTOSTUDIO Limitada de la ciudad de Villavicencio. Según datos de la Cámara de Comercio de Villavicencio (CCV, 2015) existen registradas cerca de (72) clínicas odontológicas pymes, las cuales requieren de la realización de un diagnóstico organizacional, que le permita un acercamiento real a la situación actual de las empresas. La investigación es descriptiva y como fuente primaria de información se aplicó la herramienta (MMGO) desarrollada por el Grupo de Investigación G3Pymes de la universidad EAN; esta matriz de diagnóstico es propia para pymes y permite generar un análisis más acertado a la realidad del contexto local colombiano. En cuanto al análisis de las matrices DOFA definida por la empresa y la obtenida a partir del modelo (MMGO), se observa que existe una fuerte relación entre cada uno de los componentes contemplados para cada categoría de análisis. Por un lado, se determinan como aspectos positivos la gestión humana y la planificación estratégica; y como negativos, el poco capital de trabajo que limita el apalancamiento de inversiones en renovación de equipos y la transferencia de conocimiento en nuevos procedimientos.

Palabras clave. Gestión, Modelo de modernización, Administración estratégica, planeación estratégica.

\begin{abstract}
In the current context of globalization, businesses must be at the forefront of organizational changes to improve productivity and competitiveness. The purpose of applied research is to compare the results of the diagnostic Model of Modernization for the Management of Organizations matrix (MMO) with the results of the diagnostic Weaknesses, Opportunities, Strengths and Threats matrix (WOST) from the company that provides dental services "ODONTOSTUDIO"LTM from Villavicencio city. According to the Chamber of Commerce of Villavicencio $(\mathrm{CCV}, 2015)$ there are about 72 registered pymes dental clinics which require the carrying out of an organizational diagnosis that
\end{abstract}


allows them to really approach the current situation of the companies. The research is descriptive and as a primary source of information the tool (MMO) developed by the research group G3Pymes from EAN university was applied. This matrix diagnostic is adequate for Pyres and allows generating a more accurate analysis of the reality of the Colombian local context. In relation to the analysis of the DOFA matrixes defined by the company and obtained from the MMO model, It is noted that there is a strong relationship between each of the components listed for each category of analysis; The determined positive aspects are human management and strategic planning, and the negative aspects are the little working capital which limits the leverage investments in equipment renewal and transfer of knowledge into new procedures..

Keywords. Management, management, Strategic Planning.

Modernization model, Strategic

\section{Introducción}

En el siglo XXI las organizaciones deben cumplir con muchos retos, que les exige cada día utilizar estrategias como la diversificación y diferenciación de sus bienes y servicios. Todo esto con el fin de alcanzar un mayor crecimiento como organización, así mismo necesita satisfacer cada vez más a consumidores bien informados y exigentes en un ambiente competitivo. De hecho, ofrecer nuevos servicios con un alto valor agregado y de significativa calidad, mejorando la productividad en el servicio.

En efecto, es necesario que las organizaciones, sin importar su tamaño, tengan una buena administración estratégica que les permita crecer de una manera sostenible, de ahí la necesidad de realizar un diagnóstico a la sede principal de las Clínicas Odontológicas Especializadas ODONTOSTUDIO Limitada ubicada en la ciudad de Villavicencio. El estudio se analizará desde la perspectiva de la gerente de la organización y de la secretaria administrativa, esto con el fin de poder realizar un diagnóstico comparativo a partir de la matriz del Modelo para la Modernización de la Gestión Organizacional (MMGO) y la matriz de diagnóstico de las Debilidades, Oportunidades, Fortalezas y Amenazas (DOFA). En este sentido, la relevancia de realizar un análisis radica en la evaluación del desempeño de cada una de las variables empresariales que permitan fijar planes de mejoramiento a corto mediano y largo plazo. En este sentido, es necesario cuestionarse si ¿El Modelo de Modernización para la Gestión Organizacional permite realizar un diagnóstico que contribuya a implementar estrategias para mejorar la pyme ODONTOSTUDIO?

El análisis permitió evidenciar la situación actual de la empresa, a partir de un análisis comparativo entre de los resultados obtenidos de las dos herramientas utilizadas (MMGO) y 
(DOFA). El presente artículo aborda los siguientes apartados; un contexto teórico en donde se analizan diversos conceptos y antecedentes del estudio; consecutivamente se encuentran los materiales y métodos utilizados para el desarrollo de la investigación, donde se expone la metodología, el modelo, el tipo de investigación, la población, la muestra y los instrumentos utilizados; Finalmente se presentan los resultados más relevantes y las conclusiones derivadas del desarrollo del estudio.

\section{Contexto Teórico}

En el presente apartado se señalarán los diferentes aportes conceptuales de algunos autores sobre la Gestión, Planeación Estratégica y MMGO como se puede evidenciar a continuación.

Gestión. Según Hernández (2010) "la gestión es el proceso mediante el cual se formulan objetivos y luego se miden los resultados obtenidos para finalmente orientar la acción hacia la mejora permanente de los resultados" (Marisol, 2003, pág. 5).

De acuerdo con (Rodríguez G. , 2016, pág. 32) la gestión se define como “[...] aquellas acciones relacionadas con actividades que están dirigidas a la realización y desarrollo de objetivos que han debido establecerse con anterioridad en las organizaciones." A manera de síntesis, de acuerdo a las definiciones anteriores, se puede decir que la gestión en las organizaciones es de gran importancia porque es una herramienta de la administración que permite dirigir las acciones que se desean implementar en la organización para que esta pueda ser enfocada hacia toda la organización o hacia un área específica, buscando generar un desarrollo óptimo de los procesos y cumplir con los objetivos propuestos.

Planeación Estratégica. Toda empresa debe definir unos elementos prácticos y conceptuales que permitan transformar los procedimientos para direccionar la organización hacía la consecución de su misión y visión. Por esto, la estrategia es un aspecto clave en la toma de decisiones, porque permite fijar las metas y objetivos en un horizonte de tiempo, donde se asignan de manera óptima los recursos suficientes para alcanzar los objetivos propuestos (Eugenio, A \& Barcerna , 2012, pág. 6). Igualmente es de gran importancia entender que una organización necesita de un ejercicio de planeación de sus procesos, donde éste se realiza de una manera dinámica para poder monitorear mediante indicadores de gestión, las acciones realizadas por la empresa (Amaya, 2005, pág. 22). En particular, la planeación busca implementar principios fundamentales para la firma, debido a que se requiere de un procedimiento a través de operaciones objetivas que permitan relacionar los pasos necesarios a tener en cuenta en la medida que se presenten obstáculos en el trascurso del tiempo (Caldera, 2004, pág. 32). A manera de síntesis podemos aludir que el concepto de planeación permite establecer la elaboración de un conjunto de estrategias cuyo fin es conseguir los objetivos de la organización; la planeación es una herramienta que mediante los planes de acción que permitan diseñar la hoja de ruta de la organización en el corto y largo 
plazo; Por lo tanto, la planeación y la estrategia son herramientas indispensables para utilizar al interior de una organización con el propósito de evaluar y actuar sin dejar de lado sus metas y objetivos teniendo en cuenta los cambios en los entornos a lo largo del tiempo, es por esto que los teóricos comenzaron a proponer el concepto de planeación estratégica como un instrumento de análisis con mayores alcances y posibilidades. De acuerdo con lo anterior, tradicionalmente la planificación estratégica tiene una amplia gama de definiciones, las cuales permiten tener diferentes perspectivas, por esta razón, se llevará a cabo una aproximación desde algunas conceptualizaciones. En primera instancia, se puede entender la planificación estratégica como "una herramienta de gestión que permite apoyar la toma de decisiones de las organizaciones en torno al que hacer actual $\mathrm{y}$ al camino que deben recorrer en el futuro para adecuarse a los cambios" (Evoli, 2016, pág. 5) Ciertamente, la planeación estrategica es un instrumento que apoya la toma de mejores decisiones al interior de la organización, permitiendo ejecutar la mayor parte de actividades que tiene proyectado realizar la organización en un contexto de múltiples cambios.

Por otra parte, Sallenave (2009) afirma que

La planificación estratégica es el proceso por el cual los dirigentes ordenan sus objetivos y sus acciones en el tiempo. No es un dominio de la alta gerencia, sino un proceso de comunicación y de determinación de decisiones en el cual intervienen todos los niveles Citado en (Evoli, 2016, pág. 12)

Modelo MMGO. Durante las últimas décadas, el proceso de globalización económica ha modificado la manera en que se comportan los agentes en los mercados. En este sentido, la teoría administrativa también va evolucionando de acuerdo a los constantes cambios que se realizan en las sociedades modernas. Por esto, la implementación de modelos que permitan explicar los desarrollos recientes en los entornos empresariales es de mucha importancia, debido a que se convierten en herramientas útiles en la toma de decisiones. Con base en lo anterior, el Modelo de Modernización para la Gestión de Organizaciones (MMGO), es una propuesta para las empresas que actualmente tienen interés en construir un futuro promisorio, transformando $\mathrm{y}$ utilizando el conocimiento; incorporando y desarrollando tecnología y creando valor para todos los involucrados en ella (accionistas, empresario y su familia, trabajadores, directivos, clientes, proveedores, comunidad, gobierno, competencia, entre otros). El modelo se diseñó modularmente acorde con los estudios que una empresa típica debe superar para atravesar el camino que la lleva a convertirse en una moderna y competitiva organización, con las mejores prácticas de gestión para facilitar el alcance de sus resultados (Pérez, y otros, 2009, pág. 13).

A esto se añade, que el modelo es una herramienta muy práctica para determinar el grado de modernización en la gestión organizacional, es decir, permite evaluar el desempeño de las pequeñas y medianas 
empresas con relación a su entorno situacional. De esta manera, el modelo de modernización para la gestión de organizaciones es un método de evaluación empresarial para pequeñas y medianas empresas que propone un análisis situacional y una ruta de mejoramiento para las empresas. Basado en un enfoque sistémico y holístico para diseñar e implementar una ruta de cambio hacia la innovación de las organizaciones (Pérez, y otros, 2009, pág. 19).

Adicionalmente, el modelo MMGO planteó una metodología como una caja de herramientas prácticas que permite al empresario tomar decisiones. De esta forma, el modelo emplea varios componentes para realizar el diagnóstico y la evolución de la organización, entre los cuales se encuentran: análisis del entorno económico, dirección estratégica, mercadeo, cultura organizacional, producción, finanzas, gestión humana, exportaciones, importaciones, logística, asociatividad, comunicación

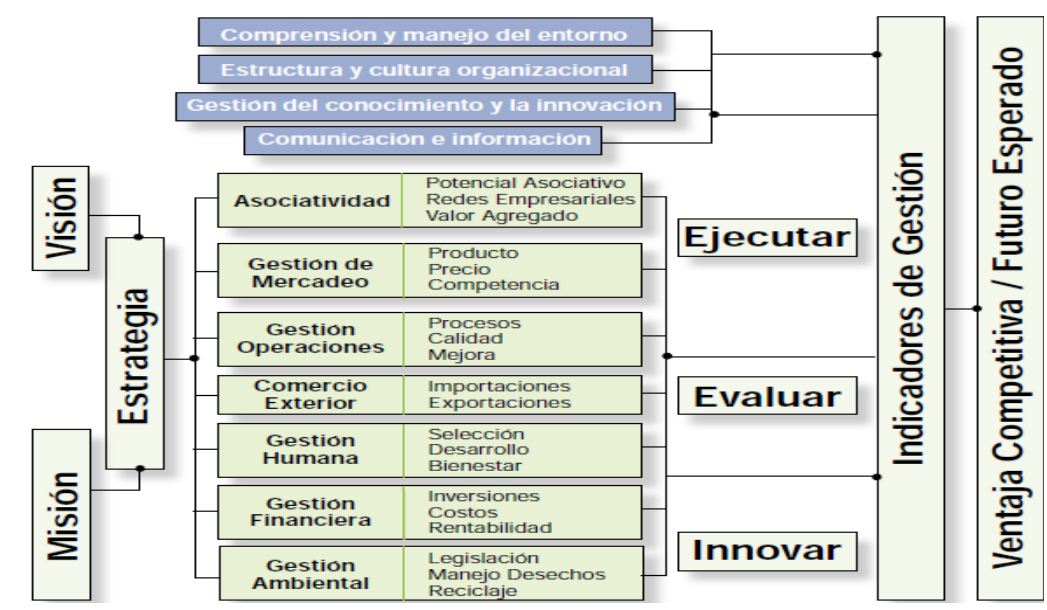

Figura 1. Componentes Organizaciones del MMGO Fuente: Nieto, Pérez, \& Velásquez, 2007. Universidad EAN. Grupo GPyMes. Recuperado de http://mmgo.ean.edu.co información, innovación y conocimiento, y medio ambiente (González, Manrique, \& González, 2010). Desde luego cada uno de los componentes esta predeterminado por variables que lo describen y que son producto de las apreciaciones en los estadios anteriores de desarrollo del proceso, donde reflejan los distintos grados por los que se enfrenta una determinada organización en la búsqueda de mejorar sus niveles de competitividad (Nieto, Pérez, \& Velásquez, 2007). Una de las fortalezas evidenciadas en este modelo de modernización y reconversión, es la capacidad de ser utilizado en empresas de diversos sectores económicos como, por ejemplo: los servicios, el comercio y la manufactura. Otra ventaja del MMGO, consiste en establecer fases en los procesos desarrollados por la empresa, que van desde la etapa de madurez hasta la identificación de las principales variables que muestran la ruta de cambio, mejoramiento y reconversión hacia una organización más avanzada (ver figura 1).

Al efectuar una investigación sobre (MMGO), se resalta la importancia que tiene su aplicación al entorno colombiano. En este sentido, (Palacio Saldarriaga, 2012, pág. 12) utiliza el modelo MMGO en la empresa INDUALCO SAS, dedicada a la producción y comercialización de alimentos, cuyo objetivo consistió en identificar las potencialidades y debilidades de la organización, para mejorar en los componentes de menor calificación.

En esa investigación, se aplicaron los catorce módulos propuestos como instrumento del MMGO los cuales son: 
análisis del entorno, direccionamiento estratégico, gestión de mercadeo, logística, comercio exterior, gestión de la comunicación y la información, innovación y tecnología, gestión humana, estructura organizacional, cultura organizacional, gestión de producción, gestión financiera, comunicación e información y responsabilidad social (Palacio Saldarriaga, 2012, pág. 9). De acuerdo con (Rodríguez T. , 2012, pág. 6), el modelo MMGO sirve para identificar y evaluar las partes críticas de la empresa, con el fin de plantear soluciones bien estructuradas, rápidas y efectivas; analizando constantemente las debilidades. Finalmente, con la aplicación de este instrumento brindarle un mejoramiento en la estructura en todas las áreas de la empresa; que sirva para implementar nuevas estrategias en los procesos para modernizar la organización.

Marco Institucional

Reseña histórica. Las clínicas odontológicas especializadas ODONTOSTUDIO Limitada, han mostrado una evolución empresarial odontológica, En el municipio de Acacias y Villavicencio, Así lo demuestra la siguiente reseña histórica de las clínicas Odontológicas

Especializadas ODONTOSTUDIO Limitada desde su creación hasta la fecha. (Ver figura 2)

ODONTOSTUDIO limitada, fue crea en el año 2006, debido a la necesidad de mejorar la práctica odontológica en el departamento del Meta, que consistió en marcar un punto de diferenciación en la prestación del servicio a través de nuevos indicadores que le permiten evaluar el desempeño en la innovación, calidad, eficiencia y efectividad en todos sus procesos, siempre tendientes a mejorar en pro de sus usuarios. Su experiencia le ha permitido consolidar una imagen corporativa en el departamento del Meta la cual es sinónimo de calidad y garantía

$\mathrm{Su}$ staff de profesionales es «Personas» idóneas, formadas bajo principios éticos y con alta experiencia en la ciencia odontológica. La capacitación constante del talento humano la innovación en los procesos y la renovación de los equipos tecnológicos son la base del éxito de la Empresa.

\section{Materiales y Métodos.}

La investigación es de tipo exploratorio, descriptivo, lo que permitió obtener información bajo modelo MMGO para determinar el estado actual de la organización clínica odontológica, ODONTOSTUDIO limitada.

La información se obtuvo a través de fuentes primarias y secundarias; como fuente primaria se aplicó el modelo MMGO; esta herramienta cuneta con 13 módulos del modelo MMGO, de los cuales se utilizaron nueve (9): el direccionamiento estratégico, la gestión de mercadeo, la logística, gestión de la comunicación y la información, el conocimiento, innovación y tecnología en la empresa, gestión humana, la estructura organizacional y la gestión ambiental; cada módulo tiene factores y estos a su vez definen indicadores de calificación de uno a cuatro; Cómo fuentes secundarias se utilizaron libros, sitio web, revistas especializadas para la obtención de investigación teórica. 

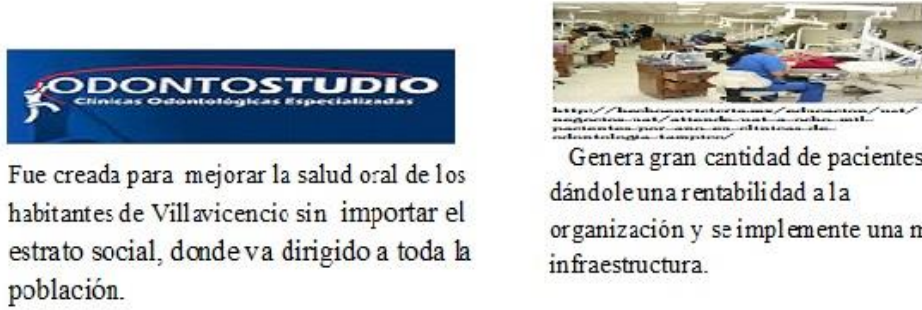

Genera gran cantidad de pacientes dándole una rentabilidad a la

organización y se implemente una mejor infraestructura.

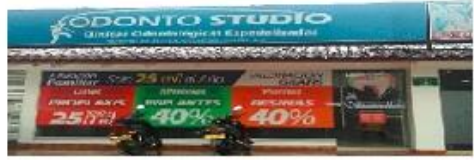

Con más de 10 años de experiencia en el mercacio logra una buena aceptación en el mercaco posicionándose en la región del Meta y devolviendo sonrisas a millones de personas.

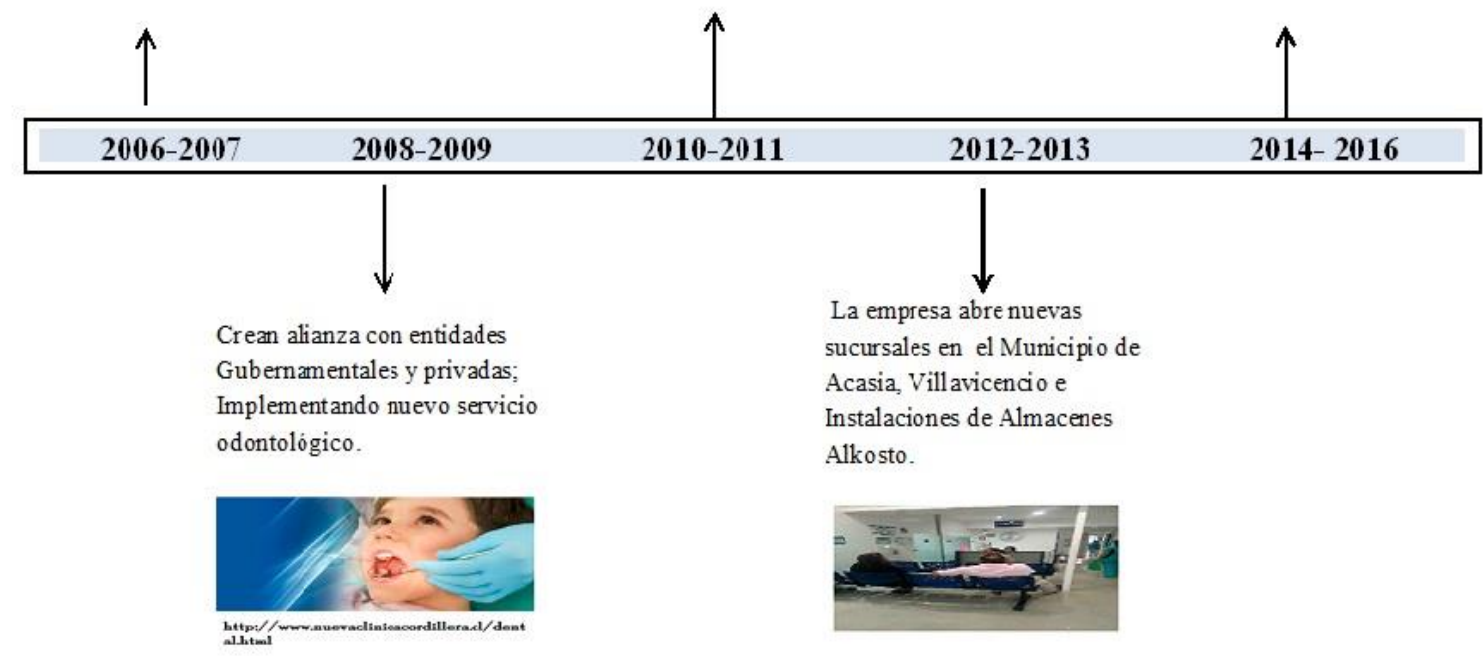

Figura 2. Línea de tiempo de la evolución empresarial de las clínicas ODONTOSTUDIO limitada. Fuente: Elaboración propia con base en información obtenida en campo

\section{Resultados}

Como se puede observar, en términos generales la empresa presenta un comportamiento positivo en los diferentes módulos contemplados en la matriz de evaluación; con una valoración promedio de $75,4 \%$.

Bajo esta premisa, y con base en las apreciaciones hechas por la gerente y la secretaria, se evidencia que los módulos de más alto desempeño son «Gestión Humana» y «Direccionamiento Estratégico», con un valoración promedio de $(90,4 \%)$ y $(89,6 \%)$ respectivamente, esto como resultado de los procesos de consolidación de una afectiva área de gestión administrativa, la cual ha tenido como finalidad el manejo de las relaciones laborales, el fortalecimiento de la cultura organizacional y la promoción de un buen ambiente laboral.

Igualmente, el fomento del conocimiento de la misión, visión y objetivos por parte de los colaboradores de la empresa han sido elementos clave para la estructuración de los planes estratégicos a corto, mediano y largo plazo. Finalmente, los componentes con más bajo desempeño, de acuerdo al análisis son: «Gestión de Mercadeo», «Estructura Organizacional», «Gestión Ambiental»y «Conocimiento, Innovación y Tecnología» con una valoración de $(69,8 \%), \quad(63,5 \%), \quad(63 \%)$ y $(62,8 \%)$ respectivamente. Esto debido a que, por un 
lado, no están definidas plenamente las estrategias de marketing que debe contemplar la empresa, situación que pone en desventaja a OdontoStudio frente a otras clínicas oncológicas de mayor reconcomiendo en el mercado gracias a su plan publicitario. Adicionalmente, la empresa no cuenta con un plan de manejo ambiental que permita definir las acciones requeridas para prevenir, mitigar, controlar y corregir los posibles efectos o impactos ambientales que puedan resultar de las acciones productivas de la organización. (Ver grafica numero 1)

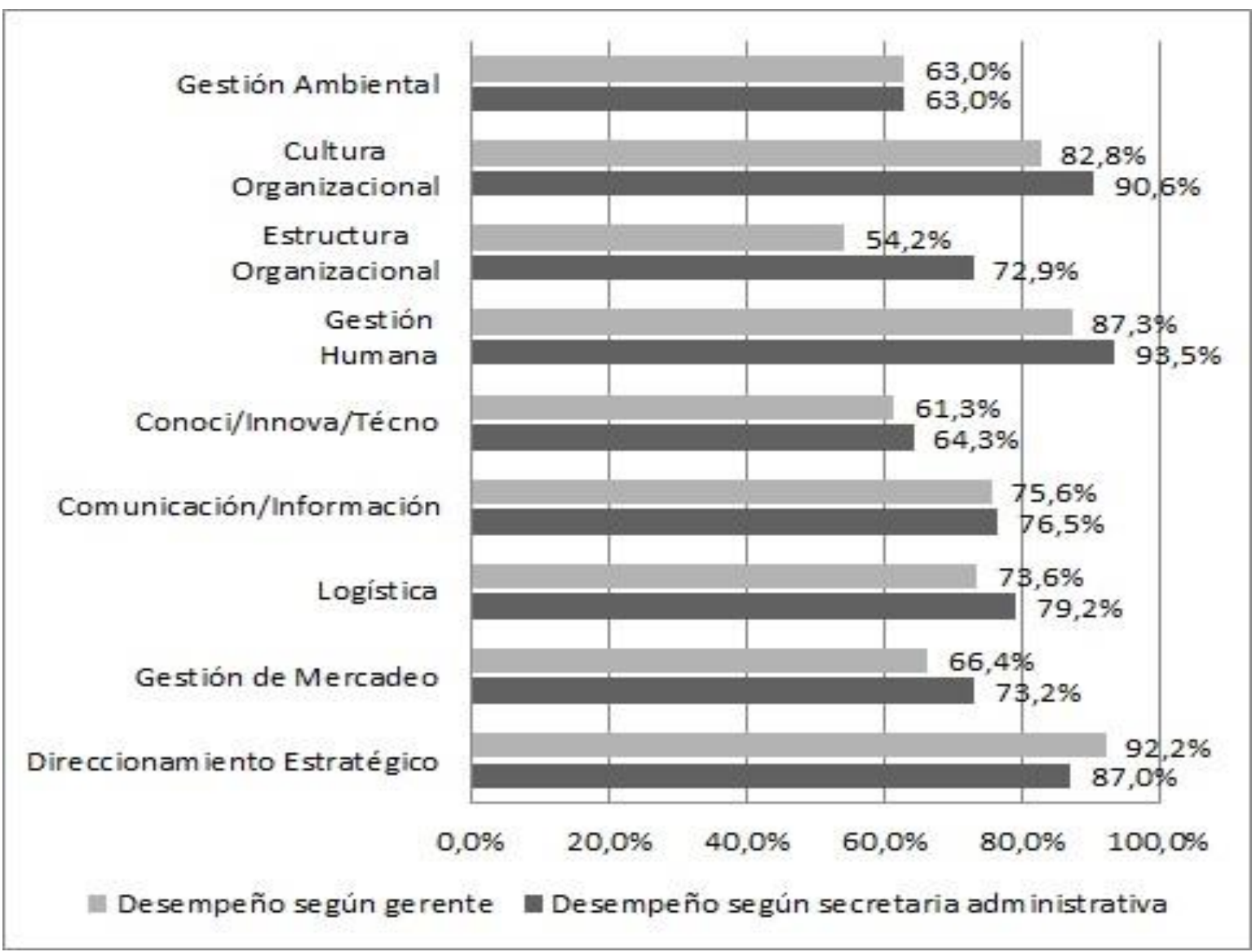

Gráfica 1. Curva de desempeño general de la Empresa, análisis comparativo según gerente - secretaria administrativa. Fuente: Elaboración propia con base en los resultados de la aplicación del instrumento MMGO a secretaria administrativa de la Empresa.

Según el objetivo propuesto de la investigación sobre la comparación de las matrices MMGO Y DOFA ORGANIZACIONAL SE EVIDENCIA QUE ENTRE LAS FORTALEZAS DE LAS DOS MATRICES EXITE una fuerte relación entre los componentes de direccionamiento estratégico, los cuales contemplan entre sus principales pilares el trabajo en equipo, los valores corporativos y la gestión de un ambiente laboral armónico. (Grafica 1 y 2). Por otro lado, el modelo MMGO señala elementos externos a nivel macro, como la estabilidad de la economía, profundización de los mercados y reglas claras en la regulación de la prestación de los servicios odontológicos, a su vez, la 
matriz DOFA establecida por la empresa, tiene en cuenta factores a nivel micro, como la asignación óptima de los recursos y la competencia a nivel de precios y calidad. Sin embargo, la restricción al capital de trabajo dificulta apalancar inversiones en renovación de equipos y conocimiento en nuevos procedimientos.
Existe, empero, una reacción adversa a los cambios institucionales que afectan el entorno del sector salud oral, como por ejemplo las resoluciones, normas y regulaciones que no cumplen con las funciones reglamentarias de protecciones al sector salud lo que hacen que el mercado no funcione de una manera eficiente.

Tabla 1. Matriz DOFA resultado del modelo MMGO

\begin{tabular}{|c|c|c|}
\hline & ANÁLISIS INTERNO & ANÁLISIS EXTERNO \\
\hline & Fortalezas & Oportunidades \\
\hline \multirow[t]{2}{*}{$\frac{2}{8}$} & $\begin{array}{l}\text { - Se cuenta con un plan de } \\
\text { gestión humana y } \\
\text { direccionami ento } \\
\text { estratégico definido a largo } \\
\text { plazo, sustentado en la } \\
\text { promoción de valores } \\
\text { corporativos y trabajo en } \\
\text { equipo. }\end{array}$ & $\begin{array}{l}\text { - Perspectivas de crecimi ento del } \\
\text { mercado. } \\
\text { - Mejoramiento tecnológico. } \\
\text { - Estabilidad de la economi a } \\
\text { nacional y regional. } \\
\text { - Estabilidad en el marco } \\
\text { regulatorio de las de servicios } \\
\text { odontológicos. }\end{array}$ \\
\hline & Debilidades & Amenazas \\
\hline 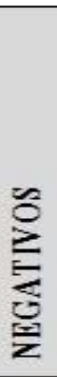 & $\begin{array}{l}\text { - Deficiencia en la } \\
\text { incorporación de } \\
\text { tecnologia, innovación y } \\
\text { conocimiento. } \\
\text { - Poco compromi so en el } \\
\text { desarrollo de la gestión } \\
\text { ambiental. } \\
\text { - Débil gestión de mercado. } \\
\text { - Resistencia a los cambios } \\
\text { en el entorno del sector } \\
\text { salud. }\end{array}$ & $\begin{array}{l}\text { - Inserción de nuevas empresas de } \\
\text { servicios odontológicos al } \\
\text { mercado local. } \\
\text { - Politicas gubernamental es que } \\
\text { desestimulan la inversión. } \\
\text { - Bajo PIB per cápita. } \\
\text { - Pagos no oportunos o no } \\
\text { realizados por parte de los } \\
\text { usuarios. }\end{array}$ \\
\hline
\end{tabular}

Fuente: Elaboración propia con base en los resultados de la matriz MMGO

Tabla 2. Matriz DOFA definida por la Empresa

\begin{tabular}{|c|c|c|}
\hline & ANÁLISIS INTERNO & ANÁLISIS EXTERNO \\
\hline & Fortalezas & Oportunidades \\
\hline \multirow[t]{2}{*}{ 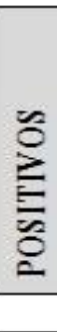 } & $\begin{array}{l}\text { - Gran experiencia en la } \\
\text { prestación de servicios de } \\
\text { salud oral. } \\
\text { - Eficiente estructura gestión } \\
\text { humana y estratégica. } \\
\text { - Calidad en el servicio y } \\
\text { precios competitivos. } \\
\text { - Solidez financiera }\end{array}$ & $\begin{array}{l}\text { - La presentación de servicios de } \\
\text { salud oral en las IPS es de baja } \\
\text { calidad y con precios altos. }\end{array}$ \\
\hline & Debilidades & Amenazas \\
\hline 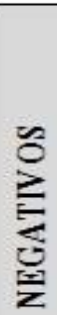 & $\begin{array}{l}\text { - Poco capital de trabajo para } \\
\text { soportar la crisis en el } \\
\text { sector salud. } \\
\text { - Elevados impuestos. } \\
\text { - Resoluciones } \\
\text { institucionales } \\
\text { Reglamentación poco } \\
\text { favorables. }\end{array}$ & $\begin{array}{l}\text { - Apertura de nueva empresas } \\
\text { odontológicas. } \\
\text { - E1 aumento en la tasa de cambio } \\
\text { restringe la adquisición de } \\
\text { herramientas y equipos de } \\
\text { última tecnologia. }\end{array}$ \\
\hline
\end{tabular}

Fuente: Elaboración propia con base en información suministrada por la Empresa 


\section{Conclusiones}

El análisis de la situación de la empresa ODONTOSTUDIO; identifica una organización sólida y definida, en cuanto a gestión humana, estrategias, cultura organizacional; igualmente, se evidenciaron debilidades en el componente de marketing digital, registrado en el módulo de conocimiento, innovación y tecnología, por tal razón este indicador tuvo un desempeño inferior. Sin embargo, esta debilidad puede solucionarse rápidamente con la disposición de recursos y tecnología para el mejoramiento del servicio. Con la ayuda de la herramienta (MMGO) se logró identificar aspectos relevantes de la estructura empresarial de la clínica odontológica ODONTOSTUDIO donde, de los 13 componentes del modelo, se pudo implementar 9 de ellos.

Al comparar el resultado de la aplicación de la herramienta de la secretaria administrativa y la gerente se observa una diferencia del $4.9 \%$ en el nivel de desempeño arrojado por la prueba, dicha diferencia se debe a la mayor calificación de los módulos de gestión humana y cultura organizacional que dio la secretaria; $(77.8 \%)$ corresponde al conocimiento de la secretaria administrativa y el $(72.9 \%)$ a la gerente. A manera general, los componentes del modelo con mayor participación promedio son la gestión humana, direccionamiento estratégico y la cultura organizacional con $90.4 \%, 89.6 \%$ y $86.7 \%$ respectivamente, mientras los de menor desempeño fueron la gestión ambiental, el conocimiento, la innovación y la tecnología y la gestión de mercadeo con un comportamiento promedio de $63 \%, 62.8 \%$ y $69.8 \%$ para cada componente.

Finalmente, dadas las implicaciones que tiene la matriz DOFA para este estudio, se hizo necesario realizar un análisis de las fortalezas, oportunidades, debilidades y amenazas que presenta la clínica odontológica ODONTOSTUDIO Limitada. Las principales fortalezas de la empresa son: el trabajo en equipo, los valores corporativos y la gestión de un ambiente laboral armónico. Así mismo, la matriz DOFA establecida por la empresa para el caso de las oportunidades tienen en cuenta factores a nivel micro como: la asignación óptima de los recursos y la competencia a nivel de precios y calidad, y a su vez, profundizar en el mercado con la realización estudios de marketing. Sin embargo, el trabajo también señaló ciertas debilidades y amenazas para la organización en los cambios institucionales que afectan el entorno del sector salud oral, como por ejemplo las normas y regulaciones que no cumplen con las funciones reglamentarias de protecciones al sector salud lo que hacen que el mercado no funcione de una manera eficiente.

\section{Referencias}

Amaya, J. A. (2005). Gerencia: Planeación \& Estrategia. Universidad Santo Tomas de Aquino. Obtenido de https://books.google.es/books?hl=es\&lr=\&id=8F lzg6f8dOsC\&oi=fnd \&pg=PA39\&dq $=+$ concepto + de+planeacion+\&ots $=\mathrm{g} 5 \mathrm{kC} \_I V K N G \& s i g=V \mathrm{c}$ VK3oRVfhmHqRgHZy6gVlufU4g\#v=onepage $\& \mathrm{q}=$ concepto $\% 20 \mathrm{de} \% 20$ planeacion $\& \mathrm{f}=$ false

Caldera, M. R. (2004). Planeación estratégica de Recursos Humanos: conceptos y teoría. España: B - EUMED. Retrieved from 
http://www.ebrary.com. Obtenido de http://bbibliograficas.ucc.edu.co:2063/lib/ucoope rativasp/reader.action?docID $=10584272 \& p p g=4$

CCV. (2015). Cámara de Comercio de Villavicencio.

Eugenio, A , K. L., \& Barcerna , J. S. (2012). Planeación Estratégica. Por Áreas funcionales. México: Alfaomega Grupo Editor.

Evoli, J. (02 de febrero de 2016). . (s.f). bbibliograficas.ucc.edu.co. Obtenido de bbibliograficas.ucc.edu.co/:http://bbibliograficas. ucc.edu.co:2063/lib/ucooperativasp/reader.action ?docID=10316642\&pp9

González, J., Manrique, O., \& González, O. (2010). La Visión Gerencial como Factor de Competitividad. Revista EAN (69), 42-59.

Great Place to Work® EE.UU. (2014). ¿Por qué la Cultura Organizacional es clave para el éxito del negocio? Obtenido de http://www.greatplacetowork.com.co/publicacio nes-y-eventos/blogs-y-noticias/740-por-que-lacultura-organizacional-es-clave-para-el-exitodel-negocio

Marisol, P. C. (14 de Septiembre de 2003). El sistema de control de gestión. Conceptos básicos para su diseño. Obtenido de http://www.gestiopolis.com/sistemacontrolgestion-conceptos-basicos-diseno/

Nieto, M., Pérez, R., \& Velásquez, A. (2007). Rutas para modernización empresarial de las Pyme. Bogotá: Publicación de la vicerrectoría de investigaciones-Universidad EAN.

Palacio Saldarriaga, M. R. (2012). Rutas para la modernización empresarial de las pymes. Obtenido de http://mmgo.ean.edu.co

Pérez, R., Nieto, M., Velázquez, A., Castellanos, G., Garzón, M., Alfonso, H., . . . López, J. (2009). Modelo de Modernización para la Gestión de Organizaciones. MMGO. Bogotá: Universidad EAN.

Rodríguez, G. (2016). Obtenido de http://www.bdigital.unal.edu.co/6678/1/claramar iagarzonrodriguez.2011.pdf

Rodríguez, T. (2012). Modelo de modernización basada en el diagnóstico de MMGO para hergo. Obtenido de http://repository.ean.edu.co/bitstream/handle/108 82/4227/NaffahLady2012.pdf?sequence $=4 \&$ is Al lowed $=\mathrm{y}$ 